\title{
Detection of cutaneous oxygen saturation using a novel snapshot hyperspectral camera: a feasibility study
}

\author{
Labrinus van Manen ${ }^{1 \#}$, Willem A. J. Birkhoff ${ }^{2 \#}$, Jeroen Eggermont ${ }^{3}$, Richelle J. M. Hoveling ${ }^{4}$, Philip \\ Nicklin $^{5}$, Jacobus Burggraaf ${ }^{1,2}$, Roger Wilson ${ }^{6}$, J. Sven D. Mieog ${ }^{1}$, Dominic J. Robinson ${ }^{7}$, Alexander L. \\ Vahrmeijer $^{1}$, Michelle S. Bradbury ${ }^{5,8,9}$, Jouke Dijkstra $^{3 \#}$
}

${ }^{1}$ Department of Surgery, Leiden University Medical Center, Leiden, The Netherlands; ${ }^{2}$ Centre for Human Drug Research, Leiden, The Netherlands; ${ }^{3}$ Leiden University Medical Center, Division of Image Processing, Department of Radiology, Leiden, The Netherlands; ${ }^{4}$ Quest Medical Imaging BV, Middenmeer, The Netherlands; ${ }^{5}$ Department of Radiology, Memorial Sloan Kettering Cancer Center, New York, NY, USA; ${ }^{6}$ Department of Anesthesiology, Critical Care Medicine, and Surgery, Memorial Sloan Kettering Cancer Center Research, New York, NY, USA; ${ }^{7}$ Erasmus Medical Center, Center for Optical Diagnostics and Therapy, Department of Otorhinolaryngology and Head and Neck Surgery, Rotterdam, The Netherlands; ${ }^{8}$ MSK-Cornell Center for Translation of Cancer Nanomedicines, Memorial Sloan Kettering Cancer Center, New York, NY, USA; ${ }^{9}$ Molecular Pharmacology Program, Sloan Kettering Institute for Cancer Research, New York, NY, USA

Contributions: (I) Conception and design: L van Manen, WAJ Birkhoff, MS Bradbury, J Dijkstra; (II) Administrative support: None; (III) Provision of study materials or patients: L van Manen, WAJ Birkhoff, RJ Hoveling, P Nicklin, MS Bradbury; (IV) Collection and assembly of data: All authors; (V) Data analysis and interpretation: L van Manen, WAJ Birkhoff, JE Eggermont, DJ Robinson; (VI) Manuscript writing: All authors; (VII) Final approval of manuscript: All authors.

\#These authors contributed equally to this work.

Correspondence to: Jouke Dijkstra, PhD. Leiden University Medical Center, Albinusdreef 2, P.O. Box 9600, 2300 RC Leiden, The Netherlands. Email: j.dijkstra@lumc.nl; Michelle S. Bradbury, MD, PhD. Memorial Sloan Kettering Cancer Center, 1275 York Avenue, New York, NY 10065 , USA. Email: bradburm@mskcc.org.

Background: Tissue necrosis, a consequence of inadequate tissue oxygenation, is a common post-operative complication. As current surgical assessments are often limited to visual and tactile feedback, additional techniques that can aid in the interrogation of tissue viability are needed to improve patient outcomes. In this bi-institutional pilot study, the performance of a novel snapshot hyperspectral imaging camera to detect superficial cutaneous oxygen saturation $\left(\mathrm{StO}_{2}\right)$ was evaluated.

Methods: Healthy human volunteers were recruited at two participating centers. Cutaneous $\mathrm{StO}_{2}$ of the forearm was determined by a snapshot hyperspectral camera on two separate study days during occlusionreperfusion of the brachial artery and after induction of local vasodilation. To calculate the blood $\mathrm{StO}_{2}$ at each pixel in the multispectral image, spectra were selected, and fitting was performed over wavelengths ranging from 470 to $950 \mathrm{~nm}$.

Results: Quantitative detection of physiological changes in cutaneous $\mathrm{StO}_{2}$ levels was feasible in all sixteen volunteers. A significant $(\mathrm{P}<0.001)$ decrease in cutaneous $\mathrm{StO}_{2}$ levels from 78.3\% (SD: 15.3) at baseline to 60.6\% (SD: 19.8) at the end of occlusion phase was observed, although $\mathrm{StO}_{2}$ levels returned to baseline after five minutes. Mean cutaneous $\mathrm{StO}_{2}$ values were similar in the same subjects on separate study days (Pearson R2: 0.92 and 0.77 , respectively) at both centers. Local vasodilation did not yield significant changes in cutaneous $\mathrm{StO}_{2}$ values.

Conclusions: This pilot study demonstrated the feasibility of a snapshot hyperspectral camera for detecting quantitative physiological changes in cutaneous $\mathrm{StO}_{2}$ in normal human volunteers, and serves as a precursor for further validation in perioperative studies.

Keywords: Optical imaging; perfusion; oxygen saturation; hyperspectral imaging; image-guided surgery 
Submitted Jan 12, 2021. Accepted for publication Apr 15, 2021.

doi: $10.21037 /$ qims-21-46

View this article at: http://dx.doi.org/10.21037/qims-21-46

\section{Introduction}

The restoration of normal tissue function and wound healing following surgery is critically dependent on tissue oxygen saturation $\left(\mathrm{StO}_{2}\right)$ and perfusion, among other factors. Tissue ischemia and soft tissue necrosis, caused by inadequate tissue oxygenation, commonly occurs in patients with (micro)vascular diseases requiring surgical interventions. However, in the post-operative period, wound complications can arise, such as tissue necrosis that necessitates a second surgical procedure. Traditionally, surgeons use tactile and visual feedback, sometimes in combination with handheld Doppler ultrasound, for intraoperatively evaluating tissue perfusion. Nevertheless, healing complications can occur, for instance, in up to $8 \%$ and $15 \%$ of patients undergoing breast reconstructive surgery or surgical amputations (i.e., below-the-knee), respectively $(1,2)$. Therefore, additional techniques that can reliably monitor perfusion and oxygenation status in postoperative settings and accurately predict and stratify patients at-risk for delayed surgical wound healing are essential for timely surgical management and for improved quality of life.

Although, transcutaneous oxygen saturation mapping $\left(\mathrm{TcPO}_{2}\right)$ and optical coherence tomography (OCT) are techniques that could be used for $\mathrm{StO}_{2}$ monitoring, only $\mathrm{Tc}_{\mathrm{PO}}$ is currently used as a standard of care procedure. Both techniques can measure $\mathrm{StO}_{2}$ levels in vivo by detecting optical absorption coefficient differences between oxygenated and deoxygenated haemoglobin $(1,3)$. However, these techniques have a limited field of view and require contact with the tissue for accurate assessments. Therefore, handheld cameras with a larger field of view, but not requiring direct skin contact, may serve as a practical alternative, in addition to being readily implemented in the perioperative setting.

One emerging technique is hyperspectral imaging, which has been used for biomedical applications, including the assessment of tumour margins and detection of tissue $\mathrm{StO}_{2}$ in pathologic conditions (4-6). This device detects the skin diffuse reflectance spectrum of light reflected from tissues after it has been scattered and absorbed by tissue chromophores, such as haemoglobin, fat, or water illuminated with a full spectrum light source (4). Current available camera systems are mostly push-broom or band sequential scanners, which are limited by the long acquisition times needed to sequentially acquire spectral wavelength bands, precluding real-time imaging evaluations. Therefore, snapshot hyperspectral cameras have recently evolved to address this drawback. The first in vivo application of such a snapshot hyperspectral camera system was demonstrated by Gao et al. for the detection of retinal $\mathrm{StO}_{2}$ levels $(7,8)$.

We have developed a novel snapshot hyperspectral imaging camera with a large field of view as a prototype system for non-invasively interrogating superficial anatomic structures and for monitoring changes in microvascular perfusion and oxygenation. This feasibility study herein aims to explore the novel capabilities of this hyperspectral snapshot camera system for detecting quantitative physiological changes in cutaneous $\mathrm{StO}_{2}$ in healthy human volunteers under ambient light conditions, and without the need for direct skin contact.

\section{Methods}

\section{Study design}

This study was an open observational multicenter feasibility study at the Leiden University Medical Center (LUMC), The Netherlands, and Memorial Sloan Kettering Cancer Center (MSKCC), USA. Healthy human volunteers, equally divided between these Centers, were recruited for participation in this study. Informed consent was obtained from all patients. All patients had a normal Body Mass Index (between 18.5 and $29.9 \mathrm{~kg} / \mathrm{m}^{2}$ ) (9), were not taking medications, and had no past medical history. The study exclusion criteria were hypertension, regular tobacco use, and any confirmed allergies to medications. Furthermore, alcohol and caffeine consumption were prohibited within 12 hours of initiating each imaging session $(10,11)$. The study was conducted in accordance with the Declaration of Helsinki (as revised in 2013). Approval for conducting the clinical trial was obtained at both Centers from their Institutional Review/Regulatory Boards. This trial was registered at the Dutch Trial Register (NL6381). All 
Table 1 Demographics of study population and overall results of cutaneous oxygen saturation measurements

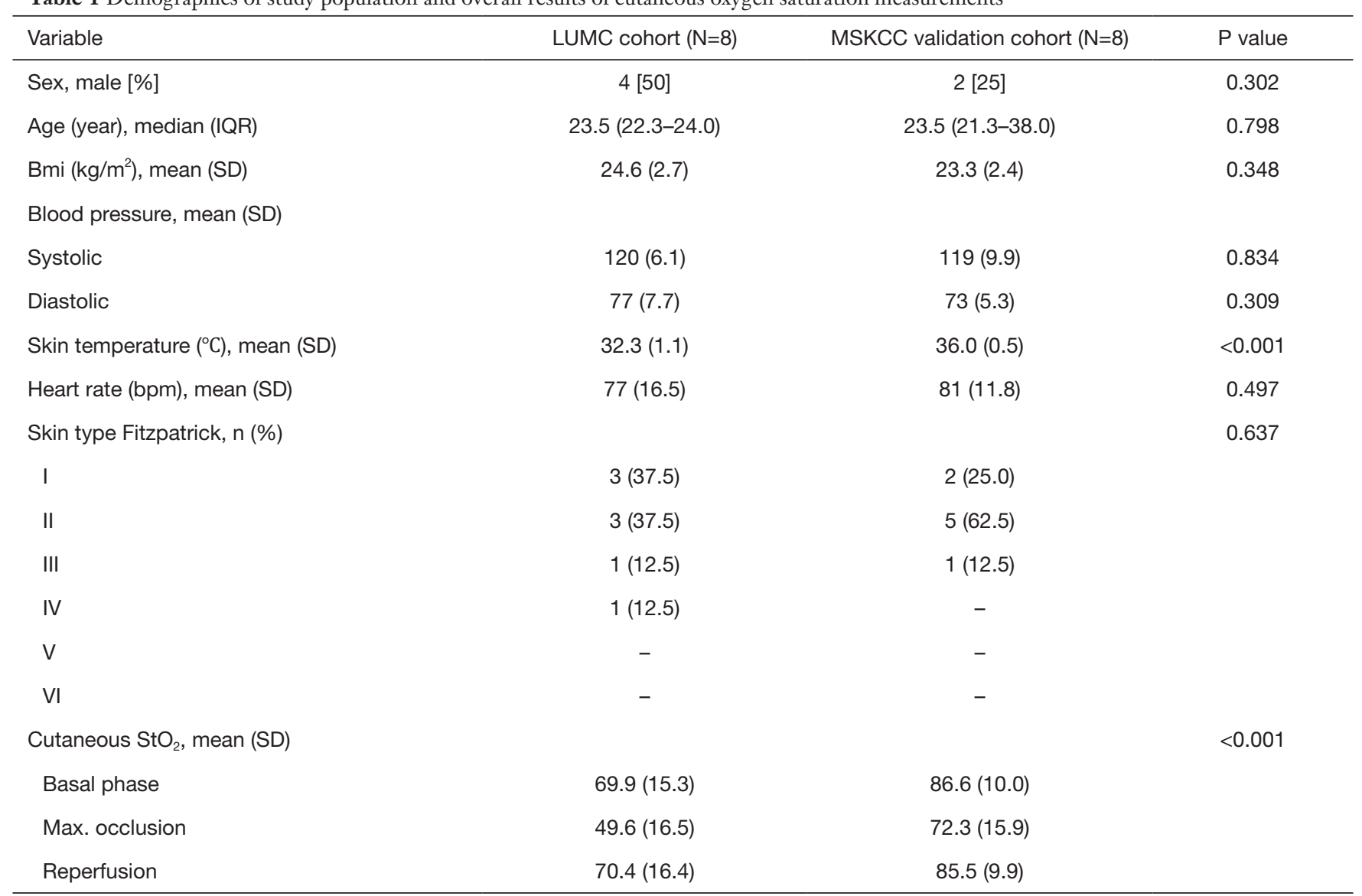

IQR, interquartile range; SD, standard deviation; $\mathrm{BMI}$, body mass index; $\mathrm{BPM}$, beats per minute; $\mathrm{StO}_{2}$, oxygen saturation.

patients provided informed consent.

\section{Study population}

Sixteen human volunteers, equally divided between trial sites, were included in this feasibility study. Subject demographics are provided in Table 1. Age, vital signs (basal blood pressure, heart rate), and the BMI, were in the range of those measured in normal healthy adults, and were similar in both patient cohorts. Skin temperatures between cohorts showed statistically significant differences [32.2 (1.1) versus $\left.36.0(0.5){ }^{\circ} \mathrm{C} ; \mathrm{P}<0.001\right]$. Skin types, assessed according to the Flitzpatrick scale, were similar between the two groups $(\mathrm{P}=0.637)$.

\section{Hyperspectral imaging system}

A snapshot hyperspectral camera system (Hyperea, Quest Medical Imaging B.V., Middenmeer, The Netherlands), which acquires 41 spectral bands across the visual (VIS) and near-infrared (NIR) spectrum, ranging between 470 and $950 \mathrm{~nm}$, was used for image acquisition (12). The camera system consists of three sensors (RGB, VIS and NIR), which are combined to facilitate simultaneous colour and spectral imaging. The effective sensor resolution is $2,048 \times 1,080$ pixels. The spectral bands are acquired using two mosaic sensors, one $(4 \times 4$ filter pattern) having a spectral range of $470-630 \mathrm{~nm}$, and one sensor $(5 \times 5$ filter pattern) having a spectral range of 600-950 nm (Figure 1). This hyperspectral camera system is based on a tiled-filter approach where pixels are individually filtered with narrow Fabry-Pérot bandpass filters (bandwidth $\approx 12 \mathrm{~nm}$ each). The camera sensors are pixel aligned on the prism, with a maximum deviation of $1 / 3$ pixel. In this study a camera lens of $35 \mathrm{~mm}$ with an aperture size of $\mathrm{f} / 2.8$ was used. The camera allows real-time imaging at video frame rates (max. frame rate: 16 frames per second) without the need to scan in either the $\mathrm{x}$ - or $\mathrm{y}$-direction. The camera lens was $35 \mathrm{~mm}$. 
A
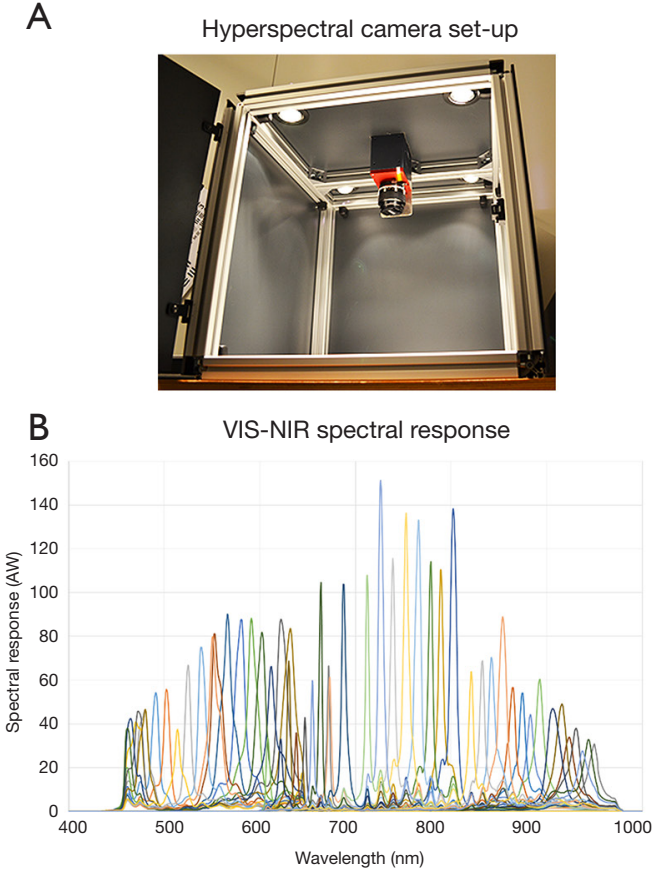

C

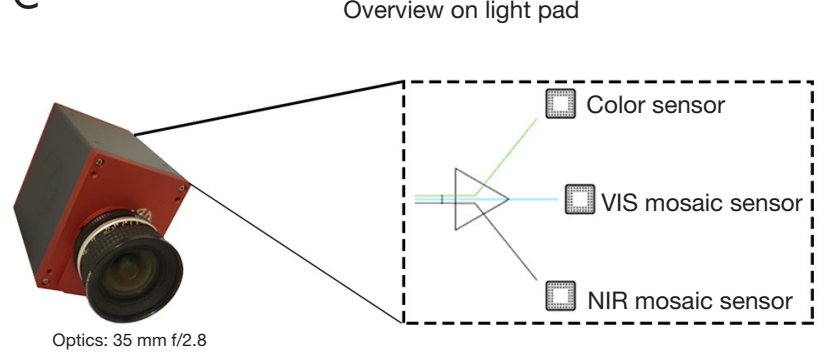

Mosaic sensors

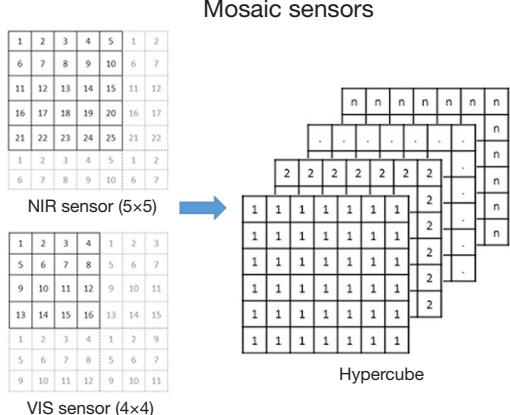

Figure 1 Overview of the hyperspectral snapshot camera system and its specifications. Images of the forearm of human volunteers were acquired by the hyperspectral snapshot camera, which was mounted in a black box containing four halogen light (broad band illumination) spots on top of each corner (A). The spectral response covers the wavelength range of 470-630 nm for the VIS sensor and 600-950 nm for the NIR sensor (B). The experimental prototype of the camera consists of three camera sensors (RGB-VIS-NIR) which are pixel aligned on the prism $(13,14)$ and a $35 \mathrm{~mm}$ lens with a $\mathrm{f} / 2.8$ aperture $(\mathrm{C})$. The camera is based on a tiled filter approach where pixels are individually filtered with narrow Fabry-Pérot bandpass filters (bandwidth $\approx 12 \mathrm{~nm}$ each). Each image acquisition leads to a hypercube with a spatial resolution of 2,048×1,080 pixels and 41 spectral bands after processing (D).

The field of view of the camera is 5 by $10 \mathrm{~cm}$ using a $40 \mathrm{~cm}$ working distance, which makes this system suitable for clinical use. A signal to noise ratio of maximum 14 could be achieved. The hyperspectral imaging set-up used for this study has four 50 Watt halogen light sources (Philips Lighting B.V., Eindhoven, The Netherlands), which were mounted on the top of a black box, for optimal control of the lighting conditions (Figure 1).

\section{Laser speckle contrast imager}

For superficial skin perfusion analysis, a Laser Speckle Contrast Imager (LSCI) (PSI; Perimed, Järfälla, Sweden) was used at one participating center (LUMC). This noninvasive technique is capable of detecting the subcutaneous movement of light-scattering particles $(15,16)$. The measurement depth of the LSCI device is approximately $0.5 \mathrm{~mm}$. The system uses a divergent laser beam with a wavelength of $785 \mathrm{~nm}$. Measurements were performed on an area of $3 \times 3 \mathrm{~cm}$, with a frequency of 22 images per second (averaged for capsaicin snapshots). Point density was normal (resolution $0.45 \mathrm{~mm}$ ). The LSCI data obtained were analysed using PIMsoft software (Perimed, Järfälla, Sweden).

\section{Imaging procedure}

To assess the imaging characteristics of the hyperspectral camera, its output was compared with contact measurements obtained using a fiber optic probe and multi-diameter reflectance spectroscopy $(17,18)$. During each measurement, the reflectance spectra are collected at effective diameters of 200, 600, and $1,000 \mu \mathrm{m} ; \mathrm{StO}_{2}$, and blood volume fraction, was determined using the largest effective single fiber diameter with an interrogation depth of approximately $500 \mu \mathrm{m}$ (19). The pathlength of light in the interrogated volume for the point spectroscopy is fixed by the fiber (equal to approximately $1 / 2$ the fiber diameter). 
For hyperspectral imaging, the illumination spot size is similar to that of the spectroscopy measurements but longer pathlength light from other points elsewhere are present in the illuminated field of the snapshot camera. Occlusionreperfusion experiments were performed to characterize tissue optical properties. In each human volunteer, baseline skin $\mathrm{StO}_{2}$ measurements were made of the right forearm over a two-minute interval. After acquiring these measurements, $\mathrm{StO}_{2}$ of the right forearm was monitored for five minutes following occlusion of the right brachial artery; this procedure was repeated during the subsequent reperfusion period. In total, the arms of the included subjects did stay for 12 minutes in front of the camera and lamps during the occlusion-reperfusion experiments. Both snapshot images and spectroscopy measurements were taken every 30 seconds, except for the first minute after reperfusion, during which phase images and measurements were taken every second.

To validate this technique, human volunteers were recruited to undergo occlusion-reperfusion at two separate times, separated by more than three days. The patients' vital signs were obtained, namely their blood pressure, heart rate, basal finger oxygenation, and skin temperature, at the start of the measurements. Occlusion-reperfusion experiments were performed, as described above. Hereafter, capsaicin cream, a vasodilator, was applied on the right forearm. It works by activating the vanilloid type- 1 receptor that, in turn, results in cutaneous vasodilation (20). Capsaicin was administered on the same arm over an area of 3 by $3 \mathrm{~cm}$, with an average distance of $5 \mathrm{~cm}$ under the radiocarpal joint, after the occlusion-reperfusion measurement. However, this was done only after the subject had rested for approximately 15 minutes, thereby restoring normal cutaneous blood flow. Subsequently, and before the capsaicin challenge, surgical tape was placed about the region of interest (ROI) to prevent spillage of capsaicin beyond the region of application. Thirty minutes after application of the crème, it was removed from the skin, and a superficial skin $\mathrm{StO}_{2}$ measurement was acquired over a 30 -minute time interval, with snapshots taken every 5 minutes. Results were compared with those of control regions located on the same forearm. In the LUMC cohort, blood flow was imaged simultaneously using LSCI.

\section{Data processing}

Due to the configuration of the $4 \times 4$ and $5 \times 5$ mosaic in the sensors and the different offsets that are applied for the placement of this mosaic pattern on the sensor pixels, specific demosaicing of the sensor information into the different spectral datacube components was required. By comparing the differences between the different planes in the spectral datacube, the alignment, cropping and scaling of the sensor data could be optimized. Based on the filter characteristics of both the sensors and the filters in the camera system, a camera-specific correction matrix could be generated for the correction of the spectral artifacts that occur due to the configuration of the filters on the sensor pixels and the nature of the interference filters, e.g., crosstalk and higher-order transmission. The correction matrix for the spectral data was validated with reference spectra (Figure 1). Next, acquired images were normalized using white and dark reference images, as previously described by Lu et al. (4). Furthermore, a correction for the second-order light effect due to light diffraction (21), provided by the manufacturer, was performed after the normalization of images. Lastly, in order to calculate the blood $\mathrm{StO}_{2}$ at each pixel in the hyperspectral images, all spectral bands $(\mathrm{N}=41)$ were selected in the full range of 470-950 nm to enable fitting using a method described previously, although implemented for hyperspectral imaging $(19,22,23)$.

As previously described, reflectance spectra were analyzed using an analytical model developed to characterize the wavelength-dependent optical properties in order to extract physiological and morphological characteristics from the tissue of interest (19). Briefly, attenuation caused by tissue absorption, is modeled using a modified Beer-Lambert law and is a function of both the tissue absorption coefficient ( $\mu$ a) and the single fiber photon path length. The scattering properties of the tissue influence both the reflectance amplitude and the single fiber photon path length, with a dependency on the reduced scattering coefficient $(\mu$ 's) and the angular distribution of scattering (phase function). The preliminary model assumed that tissue absorption was attributable to oxygenated and deoxygenated hemoglobin confined within the local microvasculature.

Minimizing the chi-squared metric between the measured reflectance data and predictions of our model was achieved by using a Levenberg-Marquardt algorithm in order to estimate the parameter values for the microvascular hemoglobin $\mathrm{StO}_{2}$, the blood volume fraction and average blood vessel diameter (19). To estimate the confidence intervals of the parameters, the square root of the diagonal covariance matrix was used (9). These values were averaged over repeated measurements, weighted by the confidence 


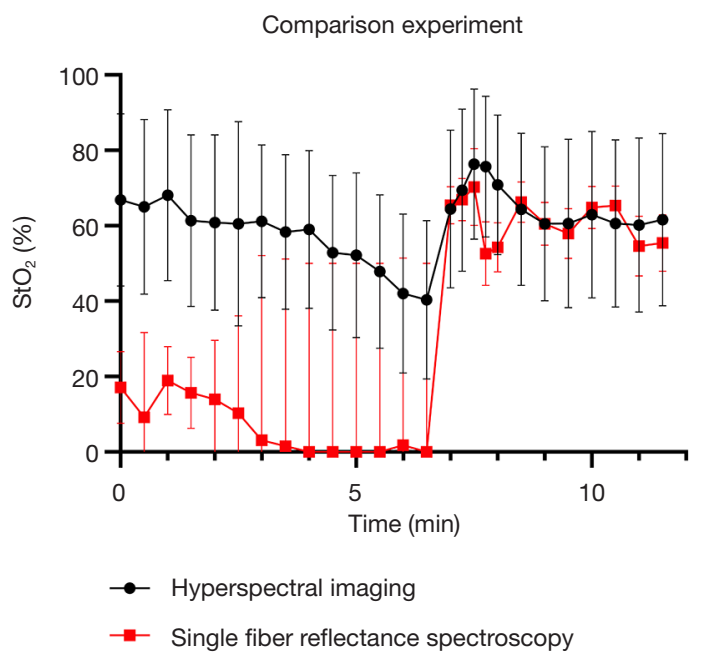

Figure 2 Comparison of single fiber reflectance spectroscopy with hyperspectral imaging for blood oxygen saturation $\left(\mathrm{StO}_{2}\right)$ detection. It shows the variation in the percentage (\%) of $\mathrm{StO}_{2}$ measured using single fiber reflectance and hyperspectral imaging. The baseline $\mathrm{StO}_{2}$ measured before occlusion using the fiber optic reflectance technique is significantly lower than that using hyperspectral imaging. During occlusion, the skin blood volume fraction is reduced. Upon reperfusion, blood volume increases and subsequently returns to near baseline levels. It is important to note that there are substantial uncertainties in both measurements of $\mathrm{StO}_{2}$ due to the relatively low blood volume in the interrogated region. $\mathrm{StO}_{2}$, oxygen saturation.

interval of individual spectral fits, and also reported with the associated weighted standard deviation.

This fitting method assumed that blood is the dominant absorber in the field-of view, and that each wavelength travels an equal pathlength in the tissue with scattering losses approximately equal across the wavelength range of interest. The wavelength dependence of the penetration depth is not a confounding factor for the spectroscopy measurements because the fiber (probe) determines the penetration depth and is approximately equal over the wavelength range measured. This is not the case for the hyperspectral camera where longer wavelength photons also contribute to the acquired signal. We determined the effective light collection area of the camera system to be approximately 320 by 320 microns per pixel. $\mathrm{StO}_{2}$ fitting was performed using software developed in-house, written in $\mathrm{C}++$ programming language. It has been shown that the recovery of blood $\mathrm{StO}_{2}$ in patients with skin types
1-3 is not affected by skin type (17). Hyperspectral data were not corrected for skin type. Saturation maps were converted to color-based maps for improved interpretation (blue: low $\mathrm{StO}_{2}$; red: high $\mathrm{StO}_{2}$ ). The fitted $\mathrm{StO}_{2}$ was not allowed to be greater than $100 \%$, nor lower than $0 \%$, for both spectroscopy and the hyperspectral camera system. The $\mathrm{StO}_{2}$ maps were imported into MeVisLab (MeVis Medical Solutions AG, Bremen, Germany), and skin $\mathrm{StO}_{2}$ subsequently calculated based on selecting corresponding ROI, which were located on a planar surface of the forearm and marked with a dermatological marker at the beginning of each measurement procedure. For all analyses, the marked area on the volar side of the forearm was chosen as the ROI. Care was taken during the repeated measurements to choose an ROI as close as possible to the previous used ROI, using the LSCI and hyperspectral images as reference. In the analysis software, the ROI was manually selected using the dermatological markers as reference point.

\section{Statistical analysis}

Continuous variables were presented as mean values \pm standard deviations (SD) in normal distributed data or as median values [interquartile range (IQR)] in non-normal distributed data, respectively. The Mann-Whitney U-test, independent $t$-test, paired $t$-test and ANOVA test were used for comparison of continuous variables. For categorical variables, a Chi-Squared test was used to compare groups. Correlations were calculated by using the mean correlation coefficient (Pearson $\mathrm{R}^{2}$ ). Correlation coefficients were interpreted according to Schober et al. (24). A P value below 0.05 (two-sided) was considered statistically significant. SPSS Statistics for Windows, version 23.0 (IBM Corp., Armonk, NY, USA) was used for the statistical analysis. Graphs were created using Graphpad 8.0 (GraphPad Software, Inc., San Diego, CA, USA).

\section{Results}

\section{Spectral fitting}

Figure 2 shows the variation in the percentage (\%) of $\mathrm{StO}_{2}$ measured using single fiber reflectance and hyperspectral imaging. The baseline $\mathrm{StO}_{2}$ measured before occlusion using the fiber optic reflectance technique is significantly lower than that using hyperspectral imaging, but is consistent with previous measurements in the skin of the inner forearm (17). During occlusion, the skin blood volume fraction is reduced 

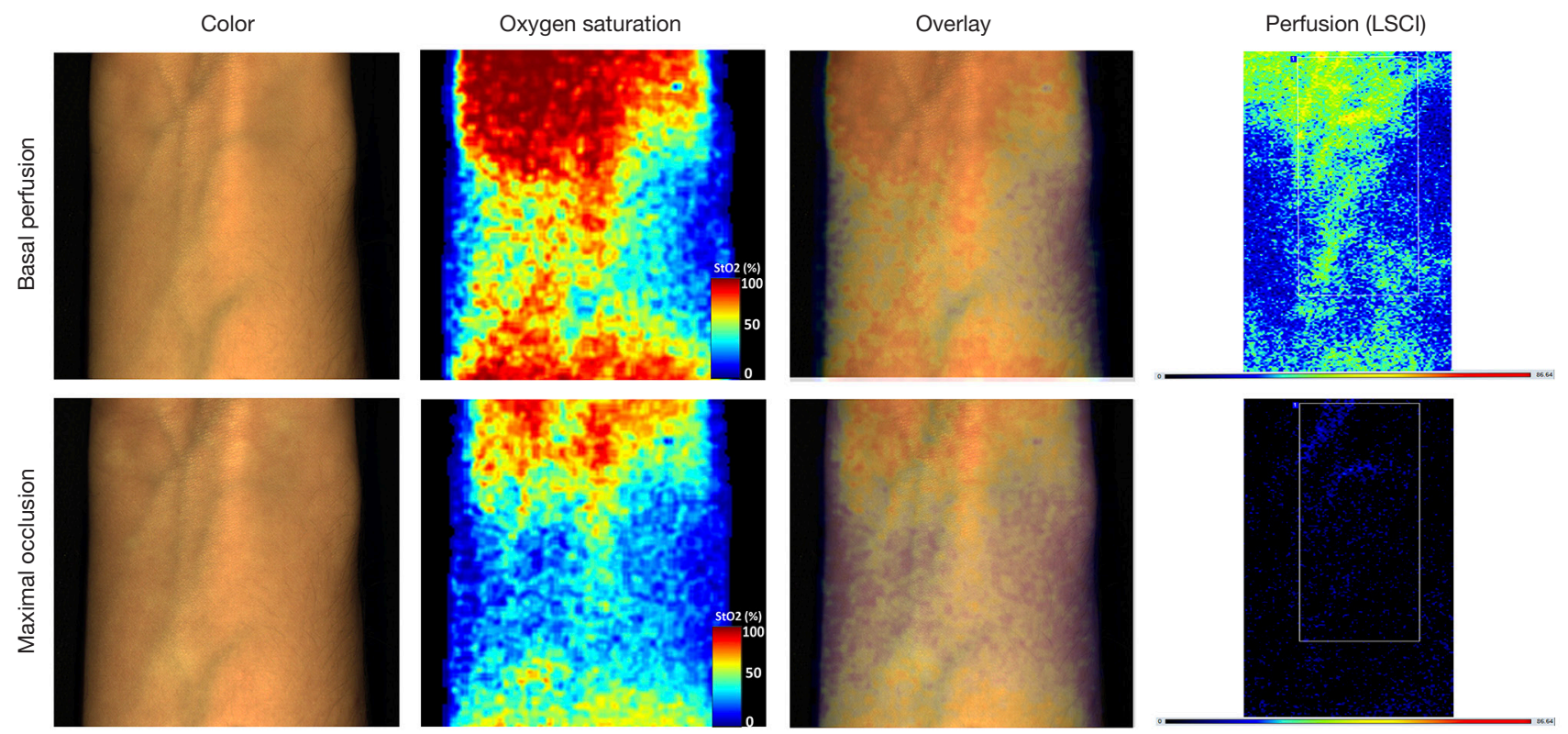

Figure 3 A typical example of the effect of vascular occlusion on superficial cutaneous oxygen saturation determined using hyperspectral imaging (color, saturation and saturation overlay maps) and perfusion determined by Laser Speckle Contrast Imaging (LSCI, perfusion maps).

(data not shown); consequently, there are large confidence intervals on these data points. Upon reperfusion, blood volume increases and subsequently returns to near baseline levels; in this regime, no significant differences in $\mathrm{StO}_{2}$ levels were found using these measurement techniques. However, it is important to note that there are substantial uncertainties in both measurements of $\mathrm{StO}_{2}$ due to the relatively low blood volume in the interrogated region, as we have described previously (9).

\section{Occlusion-reperfusion experiments}

Measurement of $\mathrm{StO}_{2}$ levels using a linear fitting algorithm was feasible in all volunteers during all phases of occlusion and reperfusion. General analysis showed a statistically significant $(\mathrm{P}<0.001)$ decrease in cutaneous $\mathrm{StO}_{2}$ levels from $78.3 \%$ (SD: 15.3 ) at baseline to $60.6 \%$ (SD: 19.8 ) at the end of occlusion phase (Figure 3). Subgroup analysis showed that baseline $\mathrm{StO}_{2}$ differed significantly between the subjects recruited at LUMC and MSKCC (mean $\mathrm{StO}_{2}$ : $69.9 \%$ versus $86.6 \%, \mathrm{P}<0.001$ ), with a decrease in $\mathrm{StO}_{2}$ of $20.3 \%$ and $14.3 \%$, respectively. Overall $\mathrm{StO}_{2}$ levels returned to baseline at the end of the reperfusion phase, which was after 12 minutes (mean $\mathrm{StO}_{2}: 70.4 \%$ versus $85.1 \%$, for the two cohorts respectively). An additional perfusion analysis was performed in the LUMC cohort using LSCI and showed a strong correlation (Pearson $\mathrm{R}^{2}$ : 0.86) of blood flow with $\mathrm{StO}_{2}$ levels during occlusionreperfusion phases (Figure 4).

Moreover, $\mathrm{StO}_{2}$ correlations between the two measurement days were analysed, resulting in an overall Pearson $\mathrm{R}^{2}$ of 0.85 , although a higher correlation was observed in the LUMC cohort (Pearson $\mathrm{R}^{2}: 0.92$ ) compared to the MSKCC cohort (Pearson $\mathrm{R}^{2}$ : 0.77). Individual analyses of all patients showed an excellent correlation in 13 $(81 \%)$, a strong correlation in $2(13 \%)$ and a fair correlation in $1(6 \%)$ subject.

\section{Effect of local vasodilatation}

After application of capsaicin cream, statistically significant increases in blood flow due to vasodilatation $(\mathrm{P}<0.001)$ were measured (with LSCI) within the most superficial layers of the skin; blood flow increased from 76.7 (SD: 11.1) to 146.9 (SD: 38.4) arbitrary units (AU). However, cutaneous $\mathrm{StO}_{2}$ did not change significantly $(\mathrm{P}=0.927)$ after application of capsaicin in the most superficial layers (Figure 5). Nevertheless, mean $\mathrm{StO}_{2}$ levels in the MSKCC cohort were significantly $(\mathrm{P}<0.001)$ higher compared to the LUMC cohort. 


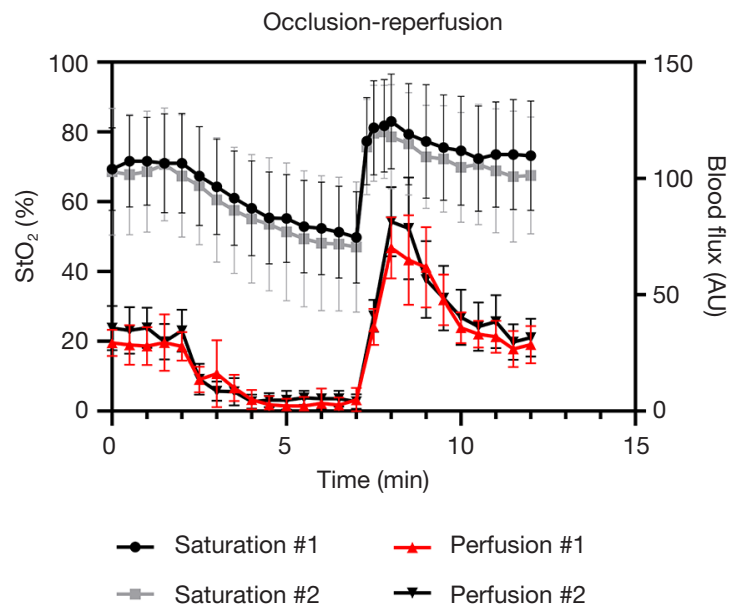

Figure 4 Correlation of cutaneous blood oxygen saturation with vascular perfusion during occlusion-reperfusion experiments in the LUMC cohort. Average of separate measurements of cutaneous blood oxygen saturation on measurement day 1 and 2 show a strong correlation with corresponding perfusion assessments by laser speckle imaging during occlusion-reperfusion experiments. Occlusion was performed after two minutes baseline measurements for a total of five minutes. Both oxygen saturation and blood flow levels returned to baseline after 12 minutes. AU, arbitrary units; $\mathrm{StO}_{2}$, oxygen saturation.

\section{Discussion}

In this multicenter study, we explored the feasibility of a novel snapshot hyperspectral camera system for noninvasive detection of superficial cutaneous $\mathrm{StO}_{2}$ under different physiological conditions in normal, healthy human volunteers. Strong correlations were found between the $\mathrm{StO}_{2}$ measurements acquired during occlusion-reperfusion experiments. Furthermore, local vasodilation did result in an increased cutaneous vascular flux, but not in a significantly higher tissue $\mathrm{StO}_{2}$ level in the most superficial layers of the skin.

In general, non-invasive imaging using endogenous contrast for the detection and monitoring of cutaneous $\mathrm{StO}_{2}$ levels is advantageous, and may offer distinct benefits over methods requiring injection of exogenous contrast agents for specific indications. In the perioperative setting, there is a general lack of real-time non-invasive imaging tools for reliably monitoring alterations in perfusion and oxygenation status. In the future, such tools could potentially improve patient care outcomes by their ability to detect decreased $\mathrm{StO}_{2}$ about surgical margins, wound sites, and sites of graft

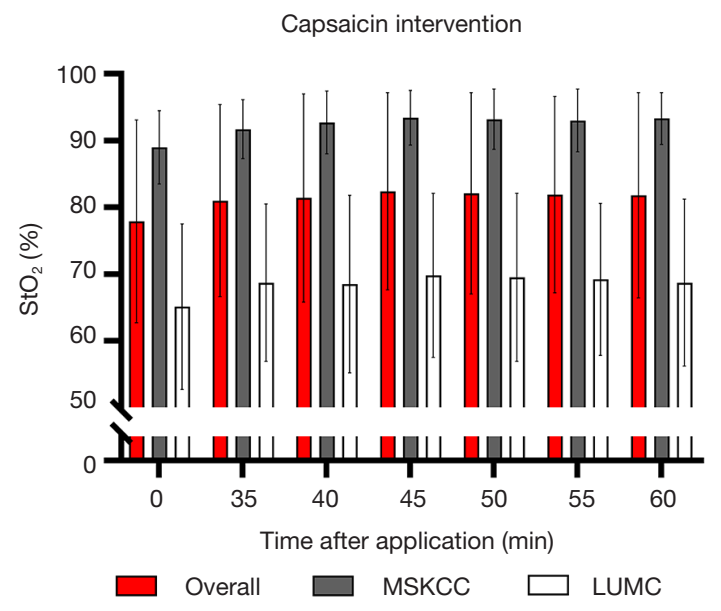

Figure 5 Tissue oxygen saturation levels after application of capsaicin cream, including subgroup analysis per cohort (LUMC vs. MSKCC validation cohort). Cutaneous $\mathrm{StO}_{2}$ levels did not change significantly after application of capsaicin in the most superficial layers. MSKCC, Memorial Sloan Kettering Cancer Center; LUMC, Leiden University Medical Center; $\mathrm{StO}_{2}$, oxygen saturation.

placement, for instance, in addition to guiding clinical management. Moreover, direct skin contact is not needed for such measurements. To our knowledge, this is the first study implementing an existing and validated algorithm for quantitatively analysing cutaneous $\mathrm{StO}_{2}$ by utilizing a snapshot hyperspectral camera system in human subjects. Recently, more systems have been introduced into the clinic for $\mathrm{StO}_{2}$ monitoring of the bowel, skin and cerebral cortex (25-29). Current available hyperspectral camera systems have longer acquisition times than those used by this snapshot hyperspectral camera system, precluding the types of evaluations performed herein. Moreover, other camera systems lack the ability to quantitatively monitor changes in tissue $\mathrm{StO}_{2}$ by using relative oxyhemoglobin or deoxyhemoglobin measurements as surrogate markers for tissue $\mathrm{StO}_{2}$. Our data supports the hypothesis that quantitative cutaneous $\mathrm{StO}_{2}$ imaging is feasible and reproducible with good correlations in human volunteers. This is also underlined by the fact that the performance of this system was independently evaluated at two different centers.

However, a few limitations are noteworthy. First, as the study recruited a relatively small number of normal, healthy human volunteers, the results may not be representative of larger patient populations, who may exhibit a variety of 
baseline characteristics. Second, the hyperspectral camera system has 41 wavelength bands divided over two mosaic sensors, which implies a lower spatial resolution (i.e., either 2 or 2.5 times) that of the original resolution, noted earlier, although this was found not to be a limiting factor in the current study. Furthermore, we decided to use contact point optical spectroscopy as a reference for comparing the $\mathrm{StO}_{2}$ of the most superficial layers of tissue. Based on these comparisons, a fitting algorithm was proposed, in order to quantify $\mathrm{StO}_{2}$, which has been validated in other clinical studies (23). Nevertheless, we acknowledge that other reference hyperspectral imaging devices suitable for $\mathrm{StO}_{2}$ detection could have also been used to supplement the perfusion data obtained by LSCI, which was unfortunately only performed one center. Moreover, a general drawback of hyperspectral imaging is that penetration depths of only several hundred micrometres to a maximum of multiple millimetres can be interrogated, which are wavelengthdependent, limiting the applicability of this optical tool to assessments of the most superficial layers of the target tissue of interest (up to the dermis at the red end of the spectrum) (30). Nevertheless, compared to contact point spectroscopy, it seems that the sampling depth is greater for the hyperspectral camera, resulting in higher $\mathrm{StO}_{2}$ values (Figure 2). The simple linear model of light transport in tissue we have used in the present study is based on assumptions that the scattering remains constant, and that the pathlength traveled is equal for all photons over the wavelength range of interest. The reduced scattering coefficient is known to vary across the visible spectrum. This means that the calculated absorbance of the tissue may be overestimated at the red end of the spectrum. Furthermore, the algorithm did not account for the effect of melanin, as it is very difficult to separate the influence of scattering and the absorption due to melanin without measuring in the bluegreen portion of the visible spectrum. Finally, alterations in penetration depth and sampling volume may occur during occlusion, given that the distribution of absorbers changes. While these changes were not accounted for in our analysis, it was felt that the scattering properties of tissues in the forearm would not be significantly affected by occlusion remote from that site.

A high inter-patient variability in cutaneous $\mathrm{StO}_{2}$ values was observed in this study, both within and across patient cohorts, however the differences in $\mathrm{StO}_{2}$ values were not significant between the two measurement days in both cohorts, which was also demonstrated by a Pearson $\mathrm{R}^{2}$ value of 0.85 . Controversial data has been published for baseline cutaneous $\mathrm{StO}_{2}$ values in the range of $50-96 \%$, depending on the camera system used and region of interest in which the measurement was taken (29,31-34). One explanation for the differences in $\mathrm{StO}_{2}$ within cohorts may be attributed to gender differences. For example, in the LUMC cohort, females tended to have a higher baseline $\mathrm{StO}_{2}$ or more subcutaneous fat. It has been demonstrated that fatty tissues are relatively poorly oxygenated, although the $\mathrm{BMI}$ included for all volunteers was within the normal range $(9,35)$. A high inter-patient variability may also be attributed to known wavelength-dependent and depth-dependent variations in cutaneous $\mathrm{StO}_{2}$ values, that is, superficial skin layers exhibit lower $\mathrm{StO}_{2}$ values than those found for the well-perfused deeper tissue layers. Another possible explanation is that skin temperature might have led to differences in cutaneous $\mathrm{StO}_{2}$ between the two cohorts, as this parameter was significantly different at baseline. The large differences in baseline $\mathrm{StO}_{2}$ between both cohorts could probably be explained by the room temperature, which was significantly higher at MSKCC, leading to increased vasodilatation with subsequent higher $\mathrm{StO}_{2}$ levels, as previously demonstrated (33). Moreover, the skin temperature could have been affected by the heat of the light sources, although $\mathrm{StO}_{2}$ levels in both groups were similar at the beginning and at the end of the measurement period. Interestingly, capsaicin interventions did not result in significantly higher $\mathrm{StO}_{2}$ levels for either patient cohort. Through activation of the TLPRV1 receptor, capsaicin is known to cause an increased neurogenic vasodilation (amongst other responses, such as flare, pain, heat, and cutaneous hypersensitivity). This challenge showed that a flare and increased vasodilation did not significantly influence $\mathrm{StO}_{2}$ measurements, which might be explained by the fact that healthy volunteers are already well oxygenated under normal circumstances and consequently the effect of local vasodilation is minimal. Nevertheless, there is still a trend towards higher saturation levels after application of capsaicin, however due to the expected limited effect on the $\mathrm{StO}_{2}$ and the small sample size of our study, the detected $\mathrm{StO}_{2}$ values are not changed significantly.

Besides hyperspectral imaging, other non-invasive techniques for $\mathrm{StO}_{2}$ mapping, such as OCT (near-infrared) spectroscopy, or photoacoustic imaging can be used, although these techniques have significant shortcomings, including the offering of only a limited field of view and/ or an inability to acquire imaging data, respectively $(3,36)$. Recently, another imaging technique termed Single Snapshot of Optical Properties (SSOP), which is based on 
the Spatial Frequency Domain Imaging (SFDI) principle, allows real-time quantitative imaging of tissue oxygenation $(37,38)$.

Future work will focus on developing a more userfriendly system and evaluating other sources for better controlled light conditions. Furthermore, data analysis steps and software will be improved to enable realtime intraoperative $\mathrm{StO}_{2}$ mapping, as current frame rate settings are only about 16 frames per second. Additional refinements, such as mounting the camera head, including light sources, on a stable arm, are needed to efficiently translate and implement such a prototype system into a variety of clinical settings. Importantly, $\mathrm{StO}_{2}$ measurements are expected to be similar for both elderly adults and younger individuals (23). In this proof-of-concept study, we focused on the assessment of cutaneous oxygenation status. For intraoperative applications, however, additional technical issues that may limit accuracy e.g., variations in skin thickness, would need to be addressed. In the present work, we attempted to minimize such contributions by imaging the inner aspect of the arm. Different applications and clinical conditions may necessitate specific mechanical solutions to be advanced, which will need to be evaluated and tested. These additional capabilities, however, would also enable high-resolution, full-field $\mathrm{StO}_{2}$ imaging to be combined, in the same setting, with other real-time imaging strategies, such as fluorescence imaging that utilizes nearinfrared wavelengths for the visualisation of multiple fluorophores. Such a hybrid system could also potentially allow for the simultaneous assessment of tissue $\mathrm{StO}_{2}$ and perfusion. As this camera system has only been evaluated in healthy volunteers, it will be necessary to validate its clinical utility in a more heterogenous patient population. Future implementation will therefore focus on assessing feasibility under clinical circumstances such as patients with vascular conditions arising from, for example, diabetes mellitus, or during reconstructive surgical procedures.

\section{Conclusions}

In conclusion, this early-stage multicenter clinical trial demonstrated the feasibility of utilizing a novel snapshot hyperspectral camera system for the detection of cutaneous $\mathrm{StO}_{2}$ in normal, healthy human volunteers. These findings are being used to inform next-stage camera system developments for monitoring oxygenation status in both non-surgical oncologic and peri-operative settings. In these settings, patients with vascular disease might potentially be risk-stratified for improving clinical decision-making and outcomes.

\section{Acknowledgments}

We thank Mark Ketelaars for his assistance during the measurement procedures.

Funding: This work was supported by the European Union Horizon 2020 - ECSEL Program under grant agreement number 692470 (ASTONISH project) and partially funded by Topconsortia for Knowledge and Innovation (TKI's) from the Dutch Ministry of Economic Affairs, as well as by the National Institutes of Health (1U54 CA199081-01 Centers of Cancer Nanotechnology Excellence to M.B.).

\section{Footnote}

Conflicts of Interest: All authors have completed the ICMJE uniform disclosure form (available at http://dx.doi. org/10.21037/qims-21-46). RJH is an employee/paid consultant for Quest Medical Imaging. The other authors have no conflicts of interest to declare.

Ethical Statement: The authors are accountable for all aspects of the work in ensuring that questions related to the accuracy or integrity of any part of the work are appropriately investigated and resolved. The study was conducted in accordance with the Declaration ofHelsinki (as revised in 2013). Approval for conducting the clinical trial was obtained at both Centers from their Institutional Review/Regulatory Boards. All patients provided informed consent.

Open Access Statement: This is an Open Access article distributed in accordance with the Creative Commons Attribution-NonCommercial-NoDerivs 4.0 International License (CC BY-NC-ND 4.0), which permits the noncommercial replication and distribution of the article with the strict proviso that no changes or edits are made and the original work is properly cited (including links to both the formal publication through the relevant DOI and the license). See: https://creativecommons.org/licenses/by-nc-nd/4.0/.

\section{References}

1. Arsenault KA, Al-Otaibi A, Devereaux PJ, Thorlund K, Tittley JG, Whitlock RP. The use of transcutaneous oximetry to predict healing complications of lower limb 
amputations: a systematic review and meta-analysis. Eur J Vasc Endovasc Surg 2012;43:329-36.

2. Wilkins EG, Hamill JB, Kim HM, Kim JY, Greco RJ, Qi J, Pusic AL. Complications in Postmastectomy Breast Reconstruction: One-year Outcomes of the Mastectomy Reconstruction Outcomes Consortium (MROC) Study. Ann Surg 2018;267:164-70.

3. Yi J, Chen S, Backman V, Zhang HF. In vivo functional microangiography by visible-light optical coherence tomography. Biomed Opt Express 2014;5:3603-12.

4. Lu G, Fei B. Medical hyperspectral imaging: a review. J Biomed Opt 2014;19:10901.

5. Zherebtsov E, Dremin V, Popov A, Doronin A, Kurakina D, Kirillin M, Meglinski I, Bykov A. Hyperspectral imaging of human skin aided by artificial neural networks. Biomed Opt Express 2019;10:3545-59.

6. Dremin V, Marcinkevics Z, Zherebtsov E, Popov A, Grabovskis A, Kronberga H, Geldnere K, Doronin A, Meglinski I, Bykov A. Skin complications of diabetes mellitus revealed by polarized hyperspectral imaging and machine learning. IEEE Trans Med Imaging 2021;40:1207-16.

7. Kashani AH, Kirkman E, Martin G, Humayun MS. Hyperspectral computed tomographic imaging spectroscopy of vascular oxygen gradients in the rabbit retina in vivo. PLoS One 2011;6:e24482.

8. Gao L, Smith RT, Tkaczyk TS. Snapshot hyperspectral retinal camera with the Image Mapping Spectrometer (IMS). Biomed Opt Express 2012;3:48-54.

9. Amelink A, Robinson DJ, Sterenborg HJ. Confidence intervals on fit parameters derived from optical reflectance spectroscopy measurements. J Biomed Opt 2008;13:054044.

10. Noguchi K, Matsuzaki T, Sakanashi M, Hamadate N, Uchida T, Kina-Tanada M, Kubota H, Nakasone J, Sakanashi M, Ueda S, Masuzaki H, Ishiuchi S, Ohya Y, Tsutsui M. Effect of caffeine contained in a cup of coffee on microvascular function in healthy subjects. J Pharmacol Sci 2015;127:217-22.

11. Gillespie JA. Vasodilator properties of alcohol. Br Med J 1967;2:274-7.

12. Manni F, van der Sommen F, Zinger S, Shan C, Holthuizen R, Lai M, Buström G, Hoveling RJM, Edström E, ElmiTerander A, de With PHN. Hyperspectral Imaging for Skin Feature Detection: Advances in Markerless Tracking for Spine Surgery. Appl Sci 2020;10:4078.

13. IMEC (2021, April 2). Available online: https://www.imecint.com/en/hyperspectral-imaging
14. Geelen B, Tack N, Lambrechts A. A compact snapshot multispectral imager with a monolithically integrated perpixel filter mosaic. SPIE MOEMS-MEMS. SPIE; 2014.

15. Matheus ASM, da Matta MFB, Clemente ELS, Rodrigues MLG, Valenca DCT, Gomes MB. Sensibility and specificity of laser speckle contrast imaging according to Endo-PAT index in type 1 diabetes. Microvasc Res 2018;117:10-5.

16. Birkhoff WAJ, Heuberger J, Post TE, Gal P, Stuurman FE, Burggraaf J, Cohen AF. Recombinant human erythropoietin does not affect several microvascular parameters in well-trained cyclists. Physiol Rep 2018;6:e13924.

17. Brooks S, Hoy CL, Amelink A, Robinson DJ, Nijsten TE. Sources of variability in the quantification of tissue optical properties by multidiameter single-fiber reflectance and fluorescence spectroscopy. J Biomed Opt 2015;20:57002.

18. Hoy CL, Gamm UA, Sterenborg HJ, Robinson DJ, Amelink A. Method for rapid multidiameter single-fiber reflectance and fluorescence spectroscopy through a fiber bundle. J Biomed Opt 2013;18:107005.

19. Stegehuis PL, Boogerd LS, Inderson A, Veenendaal RA, van Gerven P, Bonsing BA, Sven Mieog J, Amelink A, Veselic M, Morreau H, van de Velde CJ, Lelieveldt BP, Dijkstra J, Robinson DJ, Vahrmeijer AL. Toward optical guidance during endoscopic ultrasound-guided fine needle aspirations of pancreatic masses using single fiber reflectance spectroscopy: a feasibility study. J Biomed Opt 2017;22:24001.

20. Buntinx L, Vermeersch S, de Hoon J. Development of anti-migraine therapeutics using the capsaicininduced dermal blood flow model. Br J Clin Pharmacol 2015;80:992-1000.

21. Toivonen ME, Rajani C, Klami A. Snapshot hyperspectral imaging using wide dilation networks. Mach Vis Appl 2020;32:9.

22. Amelink A, Bard MP, Burgers SA, Sterenborg HJ. Singlescattering spectroscopy for the endoscopic analysis of particle size in superficial layers of turbid media. Appl Opt 2003;42:4095-101.

23. Middelburg TA, Kanick SC, de Haas ER, Sterenborg HJ, Amelink A, Neumann MH, Robinson DJ. Monitoring blood volume and saturation using superficial fibre optic reflectance spectroscopy during PDT of actinic keratosis. J Biophotonics 2011;4:721-30.

24. Schober P, Boer C, Schwarte LA. Correlation Coefficients: Appropriate Use and Interpretation. Anesth Analg 2018;126:1763-8. 
25. Barberio M, Longo F, Fiorillo C, Seeliger B, Mascagni P, Agnus V, Lindner V, Geny B, Charles AL, Gockel I, Worreth M, Saadi A, Marescaux J, Diana M. HYPerspectral Enhanced Reality (HYPER): a physiology-based surgical guidance tool. Surg Endosc 2020;34:1736-44.

26. Giannoni L, Lange F, Davies AL, Dua A, Gustavson B, Smith KJ, Tachtsidis I. Hyperspectral Imaging of the Hemodynamic and Metabolic States of the Exposed Cortex: Investigating a Commercial Snapshot Solution. Adv Exp Med Biol 2018;1072:13-20.

27. He Q, Liu T, Wang RK. Enhanced spatial resolution for snapshot hyperspectral imaging of blood perfusion and melanin information within human tissue. J Biophotonics 2020;13:e202000019.

28. Rubins U, Marcinkevics Z, Cimurs J, Saknite I, KviesisKipge E, Grabovskis A. Multimodal Device for RealTime Monitoring of Skin Oxygen Saturation and Microcirculation Function. Biosensors (Basel) 2019;9:97.

29. Nkengne A, Robic J, Seroul P, Gueheunneux S, Jomier M, Vie K. SpectraCam(®) : A new polarized hyperspectral imaging system for repeatable and reproducible in vivo skin quantification of melanin, total hemoglobin, and oxygen saturation. Skin Res Technol 2018;24:99-107.

30. Zhang R, Verkruysse W, Choi B, Viator JA, Jung B, Svaasand LO, Aguilar G, Nelson JS. Determination of human skin optical properties from spectrophotometric measurements based on optimization by genetic algorithms. J Biomed Opt 2005; 10:024030.

31. Yudovsky D, Pilon L. Retrieving skin properties from in vivo spectral reflectance measurements. J Biophotonics 2011;4:305-14.

32. Vyas S, Banerjee A, Burlina P. Estimating physiological skin parameters from hyperspectral signatures. J Biomed Opt 2013;18:57008.

33. Jafari-Saraf L, Wilson SE, Gordon IL. Hyperspectral image measurements of skin hemoglobin compared with transcutaneous PO2 measurements. Ann Vasc Surg 2012;26:537-48.

34. Chin MS, Freniere BB, Lo YC, Saleeby JH, Baker SP, Strom HM, Ignotz RA, Lalikos JF, Fitzgerald TJ. Hyperspectral imaging for early detection of oxygenation and perfusion changes in irradiated skin. J Biomed Opt 2012;17:026010.

35. Kabon B, Nagele A, Reddy D, Eagon C, Fleshman JW, Sessler DI, Kurz A. Obesity decreases perioperative tissue oxygenation. Anesthesiology 2004;100:274-80.

36. Li M, Tang Y, Yao J. Photoacoustic tomography of blood oxygenation: A mini review. Photoacoustics 2018;10:65-73.

37. Ghijsen M, Lentsch GR, Gioux S, Brenner M, Durkin AJ, Choi B, Tromberg BJ. Quantitative real-time optical imaging of the tissue metabolic rate of oxygen consumption. J Biomed Opt 2018;23:1-12.

38. Ghijsen M, Choi B, Durkin AJ, Gioux S, Tromberg BJ. Real-time simultaneous single snapshot of optical properties and blood flow using coherent spatial frequency domain imaging (cSFDI). Biomed Opt Express 2016;7:870-82.
Cite this article as: van Manen L, Birkhoff WAJ, Eggermont J, Hoveling RJM, Nicklin P, Burggraaf J, Wilson R, Mieog JSD, Robinson DJ, Vahrmeijer AL, Bradbury MS, Dijkstra J. Detection of cutaneous oxygen saturation using a novel snapshot hyperspectral camera: a feasibility study. Quant Imaging Med Surg 2021;11(9):3966-3977. doi: 10.21037/qims$21-46$ 